\title{
Current and Evolving Concepts on the Pathogenesis of Diverticular Disease
}

\author{
Antonio Tursi
}

Territorial Gastroenterology Service, ASL BAT,

Andria, Italy

\begin{abstract}
Background \& Aims: Diverticulosis of the colon is the most common anatomic alteration of the human colon, and it is characterized by the out-pouching of the colonic mucosa and submucosa through the muscular layer. Recurrent abdominal pain is experienced by about $20 \%$ of patients with diverticulosis, and inflammation of diverticula may lead to acute diverticulitis. In the past few years, several studies have investigated the factors predisposing or triggering diverticular disease (DD) occurrence. Moreover, new physiopathological knowledge has been acquired. The aim of this study was to review current knowledge regarding the pathogenesis of DD. Methods: A search of PubMed and EMBASE database was performed to identify articles relevant to the pathogenesis of DD.

Results: Several papers have shown that genetic predisposition, environmental factors, and colonic dysmotility are implicated in the pathogenesis of DD. More recent studies have associated specific host immune responses, gut microbiota imbalance and therefore low-grade inflammation as contributors to symptom occurrence in DD and diverticulitis.

Conclusions: Current and evolving evidence highlighted the role of genetic susceptibility, environment, colonic motility, visceral sensitivity, immune response, and microbiota in the pathogenesis of this disease. Further studies are required to identify potential targets for medical or surgical decision-making.
\end{abstract}

Key words: diverticular disease - genetic susceptibility - dysmotilility - inflammation - microbiota pathophysiology.

Abbreviations: CT: computer tomography; DD: diverticular disease; DICA: diverticula inflammation and complications assessment; ENS: enteric nervous system; GWAS: Genome wide association study; ICC: interstitial cells of Cajal; SNP: single nucleotide polymorphism; SUDD: symptomatic uncomplicated diverticular disease.

\section{INTRODUCTION}

The incidence of diverticulosis and diverticular disease (DD) of the colon is increasing worldwide, and its burden on national health systems in terms of direct and indirect costs is becoming significant [1]. Diverticulosis is commonly found in developed countries; it seems slightly more frequent in the USA than in Europe. It was believed to be a rare condition in the developing world [2], but new data indicate that the prevalence of colonic diverticulosis is generally increasing worldwide, probably because of changes in lifestyle in several populations [2].

Although intrinsic changes of the aged colon wall may clearly predispose to the occurrence of diverticulosis, several pathogenetic factors make the colon susceptible to diverticular disease (DD). Colonic diverticulosis remains asymptomatic in the majority of people, about $20-25 \%$ will develop symptoms, the so-called "diverticular disease" (DD), 20\% of them will develop symptoms without complications, experiencing the so-called "Symptomatic Uncomplicated Diverticular Disease" (SUDD), while about $5 \%$ of them will develop diverticulitis, with or without complications [3-6].

Despite the significant burden of the disease, the pathogenesis is poorly understood and several etiological factors may play a role in its onset. This paper focuses on the current and evolving concepts related to the pathophysiology of DD, which are summarized in Fig. 1. 


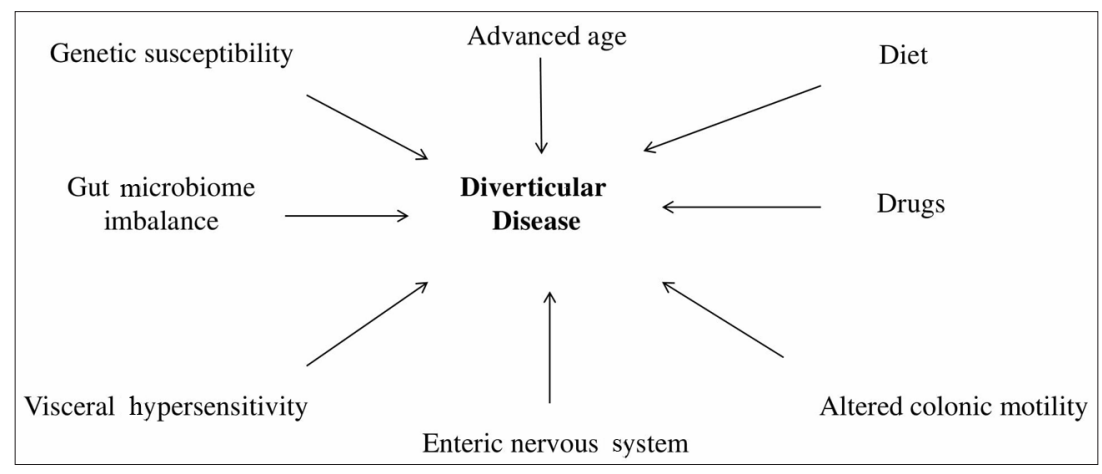

Fig. 1. Factors having a pathogenetic role in diverticular disease.

\section{GENETIC SUSCEPTIBILITY}

Two European studies investigated the role of heritability in DD occurrence $[7,8]$. The heritability of DD was estimated at $53 \%$ in a Danish twin cohort comprising twins with (923 people) and without (29,399 people) DD [7]. A comparable result was evidenced by a Swedish study, which estimated the heritability as $40 \%$ in a cohort of twins with (2,296 people) or without (102,156 people) DD [8].

More recently, new data on the role of genetic susceptibility have become available. The first single nucleotide polymorphism (SNP) associated with diverticulitis was rs7848647, a genetic variant mapping upstream of tumor necrosis factor superfamily 15 (TNFSF15) [9]. This SNP was associated with a surgical risk for diverticulitis $[9,10]$. Moreover, a rare laminin beta 4 (LAMB4) single-nucleotide variant encoding a D435N substitution was identified within a familial cluster of people suffering from diverticulitis [11]. This protein plays a role in the embryonic development of the enteric nervous system (ENS) and enhanced role of motility impairment in the pathogenesis of DD. More recently, a SNP (rs3134646) in collagen type III alpha 1 chain (COL3A1) was associated with diverticulosis in white men [12]: this result supports the historical hypothesis that an altered collagen vascular system may play a significant role in DD pathogenesis.

The first genome-wide association study (GWAS) in DD and diverticulitis was performed in 2017 in Iceland and the Netherlands [13]. A significant association with DD was found analyzing intronic variants rs4662344 and rs7609897, established within DNase hypersensitivity sites located in Rho-GTPase-activating protein 15 (ARHGAP15) and collagenlike tail subunit of asymmetric acetylcholinesterase (COLQ) [13]. rs67153654 within an intron of FAM155A (family with sequence similarity 155A) was also significantly linked to diverticulitis occurrence. Significantly, no differences were found when analyzing these SNPs on RNA expression in blood and adipocytes [13].

Finally, two large GWAS studies in DD were recently completed. The first was conducted in the United States and analyzed 27,444 cases and 382,284 controls from the UK Biobank and tested for replication in the Michigan Genomics Initiative (2,572 cases; 28,649 controls). Authors identified 42 loci associated with DD (39 of them identified for the first time), genes that are significantly enriched for expression in the mesenchymal stem cells and multiple connective tissue cell types and are co-expressed with genes that have a role in vascular and mesenchymal biology [14]. It is noteworthy that these genes in these associated loci have roles in immunity, extracellular matrix biology, cell adhesion, membrane transport and intestinal motility. The second large study was recently conducted in Europe, and reported 48 loci with GWAS. The study identified 25 previously unknown susceptibility loci for DD, 12 of which were novel: the most significant novel risk variant rs9960286 was located near CTAGE1 (cutaneous T-cell lymphoma-associated antigen 1), and the most significant novel replicated risk variant rs60869342 was located in NOV (nephroblastoma overexpressed). Based on 95\% Confidence Intervals (CIs), the authors found four loci having stronger effects for diverticulitis, namely variants at PHGR1 (proline, histidine and glycine rich 1), FAM155A-2, calcitonin-related polypeptide beta (CALCB), and the S100A10 locus [15].

Results of the latter study are particularly intriguing. These genes seem to confirm that DD is primarily a disorder of the intestinal neuromuscular function, with impaired mesenteric vascular smooth muscle function and impaired connective fiber support, whereby the risk of diverticulitis might be triggered by epithelial dysfunction. However, the impact of these genetic variants on the mechanisms of the disease remains unknown.

Table I summarizes the genetic factors associated with DD.

\section{COLONIC MOTILITY AND THE ENTERIC NERVOUS SYSTEM}

Elastosis and thickening of the colonic wall cause segmentation with non-propulsive segments, enhancing intraluminal pressure with straining of the bowel wall. This causes herniation in areas weakened by penetrating vasa recta, leading to diverticulosis [16]. Patients with diverticulosis show dysregulation of peristaltic activity, and a reduced ability of the longitudinal muscle to reach relaxation following contraction [17-19].

The sigmoid colon of DD patients shows, under basal conditions, an impaired motility (increased motility index and/ or increased contractile force) compared to healthy controls $[18,19]$. It is generally thought that dietary fiber may regulate colonic motility, but the role of fiber on this specific regulatory mechanism still requires further evidence [20]. 
Table I. Pathogenetic factors for diverticular disease occurrence

\begin{tabular}{l}
\hline Genetic susceptibility \\
Homozygous twins \\
rs7848647 (a genetic variant of TNFSF15) \\
COLQ rs7609897 \\
ARHGAP15 rs4662344 \\
FAM155A rs67153654 \\
LAMB4 D435N \\
CTAGE1 rs9960286 \\
NOV rs60869342 \\
PHGR1 \\
CALCB \\
S100A 10 locus \\
Colonic motility and enteric nervous system changes \\
Colonic structure \\
Longitudinal and circular muscle thickening \\
Elastosis \\
Segmentation \\
Alterations in the enteric nervous system (reduction of Cajal cells, S100 \\
cells and 5 HT-4R) \\
Visceral hypersensitivity \\
Low NK1 \\
Low CGRP \\
High CRLR \\
Low GDNF, RET, and GFRA1 \\
Rectal hypersensitivity \\
Brain gray matter abnormalities \\
Host immune system and pro-inflammatory findings \\
CD68 ${ }^{+}$CD163 ${ }^{+}$macrophages \\
\hline
\end{tabular}

The impairment of ENS also plays a significant role in the pathogenesis of DD. Enteric nervous system is part of the autonomous nervous system that mediates gastrointestinal control independently from the brain and spinal cord [21]. It comprises mainly two subdivisions. One is the myenteric plexus, which is located between the circular and longitudinal muscles of the colon, and comprises mostly primary afferent neurons [22]. A specific sub-population of cells, called "interstitial cells of Cajal" (ICC), represents the pacemaker cells of the gastrointestinal tract, since they mediate the input from the enteric motor nerves to the smooth muscle [23]. In the colonic ENS, we can find three subtypes of ICCs: submucosal ICCs (ICSM), located in the submucosal surface of the circular muscle layer; myenteric ICCs (IC-MY), located between the longitudinal and circular myenteric plexus; and intramuscular ICCs (IC-IM), located within the longitudinal and circular muscle layers. IC-SM and IC-MY cells generate slow wave activity, while IC-IM cells mediate input from the ENS [24]. Bassotti et al. [25] have demonstrated that a reduced number of all subtypes of ICCs, together with morphological changes and alteration of glial cells, can be found in DD patients. Enteric glial cells are important regulators of enteric neuron homeostasis, maintain intestinal epithelial barrier integrity, and may also regulate colonic motility $[26,27]$. In particular,
Immune transcriptome deregulation (RASAL3, PTPRC, INPP5D, SASH3)

Increased IL- 6 and TNF- $\alpha$

Increased IL-10

Low PGE2

Gut microbiota changes

a. Colonic microbiota

Increased:

Akkermansia muciniphila

Proteobacteria

Enterobacteriaceae

Microrobacteriaceae

Roseburia hominis

b. Colonic mycobiome

Increased Exophiala and Agaricales

c. Colonic virome

Increased human cytomegalovirus in diverticulitis

Increased expression of anti-viral response genes in earlier-onset diverticulitis

Abbreviations: 5HT-4R: 5-Hydroxytryptamine receptor 4; ARHGAP15: Rho-GTPase-activating protein 15; CALCB: calcitonin-related polypeptide beta; CGRP: galanin 1 receptor; GFRA1: GDNF family receptor alpha 1; COLQ: collagen-like tail subunit of asymmetric acetylcholinesterase; CRLR: calcitonin gene-related peptide; CTAGE1: cutaneous T-cell lymphomaassociated antigen 1; FAM155A: family with sequence similarity 155A; GDNF: glial cell-line-derived neurotrophic factor; IL: Interleukin; INPP5D: inositol polyphosphate-5-phophatase D; LAMB4: laminin beta 4; NK1: neurokinin 1; NOV: nephroblastoma overexpressed; PGE: prostaglandin E; PHGR1: proline, histidine and glycine rich 1; PTPRC: protein tyrosine phosphatase, receptor type C; RASAL3: RAS protein activator-like 3; RET: Rearranged during transfection; SASH3: Src homology-3 (SH3) domain and a sterile alpha motif (SAM) domain-containing 3; TNF: tumor necrosis factor; TNFSF15: tumor necrosis factor superfamily 15.

glial cells express the glial cell-line-derived neurotrophic factor (GDNF) that signals through the Ret Proto-oncogene (RET) to regulate colonic motility [28]. Glial cells are generally identified by $\mathrm{S} 100$, a small extension factor (EF)-hand protein containing a wide range of activity, including transmitting an extracellular neurotrophic signal to stimulate neurite outgrowth (29). A lower number of S100-positive cells has been found in the myenteric plexus in DD patients [25, 29-31]. As a consequence, GDNF, RET, and GDNF family receptor alpha 1 (GFRA1) transcripts were down-regulated in the muscular layers and myenteric ganglia of DD patients [32].

The second main mechanism of the impairment of ENS is linked to serotonin expression. We know that approximately $95 \%$ of serotonin in the body is found in the gastrointestinal tract [33], and that serotonin receptors 2 (5HT-2R), 3 (5HT$3 \mathrm{R})$ and $4(5 \mathrm{HT}-4 \mathrm{R})$ play a crucial role in initiating the peristaltic reflex and in mediating gut motility [34]. Böttner et al. [35] found recently that 5HT-4R is downregulated in the tunica muscularis of DD patients, which corresponds to the colonic wall compartment that mediates intestinal peristalsis. Moreover, authors found that $5 \mathrm{HT}-4 \mathrm{R}$ is less densely distributed in the circular muscle and myenteric plexus of DD patients [35]. This means disturbed serotonin colonic system plays an important role in causing DD occurrence. 
5HT-3R may also be a significant target for further therapeutic approaches, as confirmed by a recent pilot study [36].

Table I summarizes colonic motility and enteric nervous system factors associated with diverticular disease.

\section{VISCERAL HYPERSENSITIVITY}

Visceral hypersensitivity is another possible mechanism involved in causing symptoms in these patients. A recent work has shown that SUDD patients have visceral hypersensitivity to rectal barostat distension $[37,38]$, which also occurs in irritable bowel syndrome (IBS). In another study using rectal barostat distension, Humes et al. [39] found that SUDD patients had greater expression of neurokinin $1(\mathrm{NK} 1)(\mathrm{p}=0.01)$, as well as a higher, though not significant, expression of galanin 1 receptor (GALR1) than asymptomatic patients with diverticulosis. The increased expression of the inflammatory cytokines interleukin (IL)- 6 and Tumor Necrosis Factor (TNF)- $\alpha$, and an up-regulation of the neuropeptide receptor NK1, suggest that SUDD patients may exhibit visceral hypersensitivity due to peripheral sensitization where both inflammatory and neurochemical factors play a role.

Calcitonin gene-related peptide (CGRP) is another target in the study of the role of visceral hypersensitivity in DD. CGRP is a potent smooth muscle relaxant involved in multiple physiological processes. It acts through a heteromeric receptor constituted by a G-protein coupled receptor called calcitonin receptor-like receptor (CRLR) and a receptor activitymodifying protein 1 (RAMP1). CGRP fibers innervate many targets through the digestive system (epithelia, muscle cells, neuronal elements). Looking at the alimentary tract, CGRP exerts multidirectional action and plays a role in sensory and pain conduction, vasodilation, immune response, absorption and secretory activity [40]. Major targets of CGRP innervation are the intrinsic plexuses inducing peristaltic reflexes, increasing the peristaltic threshold, relaxing intestinal smooth muscle cells, inducing phasic contractile activity and exciting myenteric neurons [40]. Pauza et al. [37] found recently that CGRP levels within the enteric ganglia were reduced by up to $52 \%$ in symptomatic DD patients, while CRLR within the enteric ganglia were enhanced by $41 \%$ in symptomatic DD. Longitudinal smooth muscle showed an elevated (+10.5\%) relaxant effect to the exogenous application of CGRP in colonic strips from symptomatic DD patients [41]. Significantly, no consistent changes were found in patients having asymptomatic diverticulosis. This study confirms therefore, that significant alterations of nerve fiber activity may lead to a hypersensitization mechanism, that gradually takes place during DD progression.

Patients with DD have also been shown to report higher levels of somatization as measured by the Patient Health Questionnaire 12 Somatic Symptom scale (PHQ12-SS) compared to those with asymptomatic DD (ADD) [42]. This suggests that both central (psychological) and peripheral factors such as prior inflammation and changes in the enteric nervous system play a role in symptom reporting in this group [43]. Subdividing SUDD patients according to the level of somatization as assessed by the PHQ12-SS into low somatization diverticular disease (LSDD) with PHQ12-SS $<7$ and high somatization diverticular disease (HSDD) patients with PHQ12-SS > 6, Pitiot et al. [44] performed a brain analysis with a 1-mm isotropic structural brain Magnetic Resonance Imaging (MRI) scan, assessing also somatization, hospital anxiety, depression, and pain catastrophizing. Authors found a decrease in gray matter density in the left and right dorsolateral prefrontal cortex (dlPFC), and in the mid-cingulate and motor cortex, but an increase of such density in the left $[45,46]$ and right $[45,47]$ Brodmann Areas. The average cortical thickness differed overall across groups $(\mathrm{p}=0.002)$ and regionally: HSDD $>\mathrm{ADD}$ in the posterior cingulate cortex $(\mathrm{p}=0.03)$, HSDD > LSDD in the dlPFC $(p=0.03)$ and in the ventrolateral PFC $(p<0.001)$. The thickness of the anterior cingulate cortex and the mid-prefrontal cortex were also found to correlate with pain catastrophizing $(\mathrm{p}=0.03)$ [44]. This study shows for the first time that structural gray matter abnormalities may occur in SUDD patients, and that brain differences may play a significant role in the pain network of those patients.

Table I summarizes alteration of visceral sensitivity associated with DD.

\section{ENVIRONMENTAL FACTORS}

\section{a. Diet}

The keystone paper from Painter and Burkitt described the role of reduced dietary fiber in the development of DD [48]. In particular, they found the diverticulosis prevalence to be higher in Westernized people where dietary fiber intake is lower compared to African people with higher levels of dietary fiber intake [48]. Further reports confirmed this association, demonstrating that insoluble fiber intake decreased the risk of DD $[49,50]$. Similarly, Crowe et al. found that a high-fiber diet reduced the risk of hospital admissions and mortality due to DD [51, 52]. Moreover, a vegetarian lifestyle and nuts and popcorn consumption reduced the risk for diverticulitis $[51,53]$. Finally, an increased risk of diverticulitis was found in individuals with low levels of vitamin $\mathrm{D}$ and in men with a high consumption of red meat $[54,55]$.

However, these findings have been recently challenged, since an association between low dietary fiber intake and increased risk of diverticulosis occurrence was not found $[56,57]$. The evidence regarding the role of dietary fiber in DD pathogenesis remains therefore inconsistent [58], even if literature data suggest that dietary fiber supplementation may have a beneficial impact on the intestinal microbiota [59].

\section{b. Ultraviolet exposure and lifestyle}

People living in areas with low exposure to ultraviolet light or in rural areas had a higher risk of diverticulitis-associated hospitalizations than people living in areas with high ultraviolet light exposure $[60,61]$. Smoking was found to be a risk factor for complicated diverticulitis and hospitalizations [61-64]. Also obesity was associated with DD complications (diverticulitis, diverticular bleeding, hospitalization) $[60,65]$, while physical activity reduces the risk of diverticulitis and diverticular bleeding [66].

Two recent case-control studies assessed the role of alcohol and smoking in the pathogenesis of DD. Assessing these associations in a prospective cohort of 47,678 men living in 
United States, Aldoori et al. [67] found that alcohol intake (comparing those who drink $>30 \mathrm{~g}$ of alcohol/day to nondrinkers) was only weakly and non-significantly associated with the risk of symptomatic DD (relative risk $\mathrm{RR}=1.36$; 95\% CI 0.94 to 1.97 ; $\mathrm{p}$ for trend $=0.37$ ). They observed no association between caffeine, specific caffeinated beverages, and decaffeinated coffee and the risk of symptomatic DD. Current smoking was not appreciably associated with the risk of symptomatic DD compared to non-smokers $(\mathrm{RR}=1.25$; 95\% CI, 0.75 to 2.09) [67].

More recently, Nagata et al. [68] found that age, male sex, smoking index, alcohol consumption, aspirin, anticoagulants, corticosteroids, hypertension, and atherosclerotic disease were factors significantly associated with diverticulosis, and that increasing age $(p<0.01)$, increased alcohol consumption $(\mathrm{p}<0.01)$, smoking $(\mathrm{p}<0.01)$, and atherosclerotic disease $(p<0.01)$ were significantly associated factors at multivariate analysis. Alcohol and smoking were also associated with rightsided and bilateral diverticula [68].

\section{c. Medical treatments}

Medical therapy can also play a role as a risk factor for DD occurrence. Use of oral corticosteroids, opiate analgesics, and non-steroidal anti-inflammatory drugs (NSAIDs) has been associated with complicated diverticulitis [64, 69-71]. It is thought that NSAIDs induce mucosal damage through the reduction of prostaglandin synthesis [70], while the mechanism by which steroids and opioids cause complicated diverticulitis is less understood. Furthermore, the use of statin and calcium channel blockers may reduce the risk for diverticular bleeding $[64,72]$

Table II lists environmental factors, voluptuary habits and drugs associated with diverticular disease.

Table II. Environmental factors, drugs and lifestyles associated with diverticular disease

Obesity

Smoking

Vitamin D deficiency

Red meat consumption

UV light lower exposure

NSAIDs

Corticosteroids

Opioids

NSAIDs: non-steroidal anti-inflammatory drugs; UV: ultraviolet

\section{HOST IMMUNE RESPONSE AND ROLE OF INFLAMMATION}

A fundamental role in the growth and modulation of the host immune response is played by the commensal human gut microbiome, which is a dynamic ecosystem composed of bacteria, fungi, viruses, and other microorganisms. All of them live in symbiosis with the host, and modulate and promote immune tolerance [73]. Two main mechanisms are involved in maintaining host homeostasis. The first is the innate immune system, which is the first line mechanism against pathogens and promotes early activation of the inflammatory response in the intestinal tract [74]. The primary function of the innate immune system is to discriminate between pathogenic and non-pathogenic foreign antigens and maintain intestinal homeostasis by activation of phagocytic cells [75]. The second is the adaptive immune response, which is involved in developing immunological memory against pathogens, promoting an adequate response, and consequently eliminate pathogens following subsequent encounters [75]. These processes are mainly mediated by $\mathrm{T}$ and $\mathrm{B}$ cells.

\section{a. Changes in immune cells expression}

Although current thinking regarding the pathogenesis of diverticulitis is that bacterial overgrowth activates the immune system leading to mucosal inflammation, the role of the immune system in DD remains understudied.

Diverticulosis does not show changes in the immune system. Peery et al. [76] analysed recently several immune cells and cytokines by colonic mucosal biopsy specimens from people with diverticulosis. Assessing levels of interleukin-6 (IL-6), interleukin-10 (IL-10), tumor necrosis factor-alpha (TNF- $\alpha$ ), and several immune cells (CD4+, CD8+, CD27+, and mast cell tryptase), they observed no significant changes in the expression of such immune cells/cytokines in these people [76].

On the contrary, significant changes were detected in DD/ diverticulitis. Von Rahden et al. [77] found an association between activated CD68+CD163+ macrophages in the sigmoid colon of patients with complicated diverticulitis relative to those with chronic, recurrent diverticulitis. Furthermore, the high expression of CD68+CD163+ macrophages correlated with steroid intake [77]. More recently, an increased number of CD68+ macrophages was observed in SUDD patients, with no differences in mast cells or CD3+ T cells expression [78].

Patients with SUDD show also a significant microscopic inflammatory infiltrate. More than ten years ago, Narayan and Floch [79] found a significant inflammatory infiltrate in SUDD patients compared with healthy controls. We assessed both neutrophilic and lymphocytic infiltrate in SUDD, AUD and healthy controls. While neutrophilic inflammatory infiltrate was found only in AUD, the mean lymphocytic cell density was significantly higher in SUDD than in asymptomatic diverticulosis and in controls $(\mathrm{p}<0.02)$ [80].

\section{b. Changes in inflammatory mediators}

Mucosal inflammatory mediators are also deregulated in SUDD patients. TNF- $\alpha$, a $17-\mathrm{kDa}$ polypeptide produced by macrophages, lymphocytes and natural killer cells, has been shown to play a major role in the inflammatory processes, with high levels being found both in Inflammatory Bowel Diseases (IBD) patients and in rheumatoid arthritis [81-83]. Hypothesizing a possible role of low-grade inflammation in the occurrence of SUDD, TNFa was assessed in those patients. Patients with SUDD had a significant overexpression of TNF- $\alpha$ and IL- $6[84,85]$, while mucosal TNF- $\alpha$ decreased during remission [86]. Similar trends were also found for basic fibroblast growth factor (b-FGF) and syndecan-1 (SD1) [86].

IL-10 is an important anti-inflammatory mediator that suppresses the production of pro-inflammatory cytokines [87]. Surprisingly, IL-10 expression in SUDD seems to 
have an opposite effect than the one expected. Turco et al. [88] found a slight increase of IL-10 release in SUDD and a significant IL-10 release in SUDD following acute diverticulitis (SUDD-AD) compared to healthy controls $(\mathrm{HC})(\mathrm{p}<0.05)$. We recently confirmed these results. Using an Enzyme-linked immunosorbent assay (ELISA) analysis on fecal samples, we found an increased release of IL-10 in SUDD patients compared to asymptomatic diverticulosis and $\mathrm{HC}$, although no significant difference was found [89].

Thus, we can speculate that IL-10 expression in SUDD may be an attempt by the immune system to control a low-grade inflammation. This hypothesis seems to be supported by Dai et al. [90], who found an inverse expression of prostaglandin $\mathrm{E} 2$ in colonic mucosa between acute diverticulitis and IBD. We know that prostaglandin E2 is the dominant prostaglandin in the colon and is associated with colonic inflammation, but we also know that it has a protective mechanism in the mucosa of the gastrointestinal tract. Lower prostaglandin E2 level may reduce the normal protection of the mucosa, which becomes more susceptible to luminal insults, thus creating a permissive environment for the development of acute diverticulitis.

Schieffer et al. [91] found recently that both innate and adaptive immune system pathways were deregulated in diverticulitis patients compared to non-diverticulosis controls. They identified four immuno-regulatory hub genes [RAS protein activator-like 3 (RASAL3), protein tyrosine phosphatase, receptor type C (PTPRC), inositol polyphosphate-5-phophatase D (INPP5D), and SAM and $\mathrm{SH} 3$ domain-containing 3 (SASH3)] that were associated with diverticulitis. Overall, these findings suggest that DD is associated with deregulation of the immune system.

c. Relationship between host immune response, lowgrade inflammation and other mechanisms leading to symptoms' occurrence

Low-grade inflammation may also interact with other pathogenetic mechanisms to trigger the symptoms.

For example, visceral hypersensitivity has been suggested as a possible mechanism involved in causing symptoms in these patients. Humes et al. [85] found that SUDD patients had a greater expression of neurokinin 1 (NK1) than asymptomatic patients with diverticulosis $(\mathrm{p}=0.01)$, as well as a higher but not significant expression of GALR1 receptor. The increased expression of the inflammatory cytokines IL- 6 and TNF $\alpha$, and an up-regulation of the neuropeptide receptor NK1, suggests that SUDD patients may exhibit visceral hypersensitivity due to peripheral sensitization with both inflammatory and neurochemical factors playing a role.

Diverticular disease is associated with peculiar alterations of the ENS (myenteric oligoneuronal hypoganglionosis, decreased intramuscular nerve fibers, and altered neurochemical coding) [2]. Analyzing the expression of the glial markers $S 100 \beta$, Cossais et al. [92] found very recently that $S 100 \beta$ expression was increased in the submucosal and myenteric plexus of patients with DD compared to controls, while expression of other glial factors remained unchanged. Moreover, this $S 100 \beta$ overexpression was correlated to CD3+ lymphocytic infiltrates in patients with $\mathrm{DD}$, while no correlation was observed in controls.
Table I summarizes the host immune factors associated with diverticular disease.

\section{GUT MICROBIOME}

Despite the large use of empiric antibiotics in these patients, the role of commensal intestinal microbiome in DD is still poorly understood. A dual layer of mucus composed of large and highly glycosylated proteins called mucins covers the intestinal epithelial cells. Mucin 2 (MUC2) is the primary mucin secreted by the intestinal goblet cells [93]. This barrier is vital to protect the underlying tissues from invasion by commensal bacteria [94]. Additionally, the colonic epithelium expresses specific antimicrobial peptides protecting against microbial invasion [95].

\section{a. Colonic microbiota}

The human gut microbiota is a variable ecosystem, mainly represented by two distinct phyla, Bacteroidetes and Firmicutes [96]. Although it is relatively stable within an individual [97], a higher diversity is found among subjects: this indicates that the human gut microbiota is probably linked to the single individual rather than the same in the overall population [98].

Microbial imbalance, which is known as dysbiosis, has been associated with many gastrointestinal diseases, including IBD [99]. Alterations in the gut microbiota have been hypothesized as a potential etiological factor involved in the pathogenesis of DD and diverticulitis [100]. However, until now the role of the microbiota in DD has remained elusive, and results appear still controversial, particularly when compared with other wellknown diseases. For example, Roseburia hominis is decreased in UC [101] but increased in DD [102]. Moreover, Akkermansia muciniphila (A. muciniphila) was found decreased in IBD [103] and increased in SUDD [104]. However, a more recent study on patient-matched samples found an increased abundance of A. muciniphila in the unaffected descending colon in SUDD patients [78]. Finally, a recent pilot-study found that the amount of $A$. muciniphila species was significantly reduced after $30(p=0.017)$ and $60(p=0.026)$ days after the end of the treatment, while after 90 days the reduction was not significant in comparison to the enrollment $(\mathrm{p}=0.090)$ [105]. Moreover, these changes in A. muciniphila colonization were significantly linked to the symptoms' expression [105].

Why this occurs is still unknown. A. muciniphila is a mucolytic bacteria living in the mucus barrier that degrades mucin for utilization as a carbon and nitrogen energy source [106]. However, this bacterium is beneficial to the host, producing short-chain fatty acids to maintain intestinal homeostasis [107]. Depletion of A. muciniphila has been associated with IBD occurrence [103], while increased levels of $A$. muciniphila were observed in elderly people, the very population in which generally DD occurs [108]. The role of $A$. muciniphila in DD remains therefore controversial.

Several studies evaluated the gut microbiota of diverticulitis patients compared to controls. Daniels et al. [109] found that the fecal microbiome differs significantly between patients at the first episode of acute diverticulitis compared to controls, 
with the largest difference defined by a higher diversity of Proteobacteria [109]. More recently, Schieffer et al. [110] compared chronically diseased full-thickness sigmoid colon tissue to adjacent tissue and found Microbacteriaceae as the bacteria most significantly associated with the diseased segment of the sigmoid colon [110]. According to previous data, Proteobacteria were overexpressed in both tissue sections and comprised the core microbiota of these patients [110]. The association of Proteobacteria and diverticulitis could be a consequence of the inflammatory process, since an increase in Enterobacteriaceae has been associated with nonspecific experimental colonic inflammation [111].

Also, patients with prior diverticulitis seem to have specific microbial changes. Kvasnovsky et al. [112] found recently that patients with a previous episode of acute diverticulitis did not show a microbiota alpha or beta diversity, but had a higher relative abundance of Pseudobutyrivibrio, Bifidobacterium, Christensenellaceae family, and Mollicutes RF9 order. Also, persistence of symptoms seems to have a significant relationship with microbial imbalance (higher relative abundance of Ruminococcus, Roseburia, and Cyanobacterium) [112].

These studies show differences in bacterial identification methods, sample site collection, prior and concurrent therapeutic courses and utilization of control groups, all of them potentially able to confound results. Moreover, many of these studies were performed with small sample sizes, without an adequate statistical power. Thus, a specific dysbiotic microbial composition that influences DD or diverticulitis has not been identified yet.

\section{b. Colonic mycobiome}

In comparison to the gut microbiota, the human gut mycobiome, comprising the fungal communities, is still understudied [113]. Fungi are found within the gastrointestinal tract of the majority of adult population [114], and Candida is the dominating species [115]. A core mycobiome has not been established yet, but we know that the mycobiome is not stable over time [116]. Dollive et al. [116] analyzed a mice population, and found that a longitudinal variation in fungal composition could be found while the bacterial population remained stable during the observational period, suggesting that the mycobiome may be more influenced by the environment. More recently, Schieffer et al. [110] evaluated the mycobiome of diverticulitis patients by internal transcribed spacer (ITS) sequencing. Assessing matched full-thickness chronically diseased and adjacent sigmoid colon tissue, they found that an abundance of Exophiala correlated with diseased tissues, whereas an abundance of the order Agaricales was found in the adjacent tissues [110]. These are interesting but only preliminary results, and further studies are required to investigate the role of these organisms in the pathogenesis of DD.

\section{c. Colonic virome}

The human gut virome seems to play a significant role in controlling immune response and tolerance to intestinal microbiota [117]. Unfavorable alteration of gut virome composition has been implicated in chronic immune disorders, such as in the pathogenesis of IBD [118].
Analyzing serum samples for the presence of human cytomegalovirus (HCMV)-IgG and HCMV-IgM, and the intestinal paraffin tissue sections obtained from the diverticulitis patients by immunohistochemistry, Hollink et al. [119] found that HCMV-early proteins could be detected in intestinal cells in $16 / 23(69.6 \%)$ patients with diverticulitis, and that all of them with serum samples were HCMV-IgG positive. Very recently, Schieffer et al. [120] conducted a deep RNA sequencing (RNA-seq) analysis on colonic segments surgically resected from earlier-onset ( $<42$ years old) and later-onset $(>65$ years old) diverticulitis patients. Authors found that the earlieronset patients displayed an increased expression of anti-viral response genes, confirmed by using an independent weighted co-expression network analysis (WGCNA) of differentially expressed genes [120]. This preliminary report may mean that a differential host response to viral infection could define a subset of earlier-onset diverticulitis patients.

Table I summarizes gut microbiome changes associated with diverticular disease.

\section{CONCLUSIONS}

Diverticulosis is a common condition that sometimes becomes symptomatic and may lead to severe complications.

The pathophysiologic hypothesis behind diverticulosis and subsequent symptoms' occurrence in patients with diverticulosis has changed in the last years. Firstly, the already known pathogenetic role of environmental factors, colonic motility and visceral hypersensitivity has been enriched by new evidence. Secondly, novel immunologic pathways and genetic associations, as well as new data on gut microbiota are becoming available. However, most of these data are too preliminary to draw any conclusive indication for clinical practice. All these recent advances have to be therefore considered as a pathway for future research to identify possible targets for medical or surgical options.

Conflicts of interest: The author declares that he has no conflicts of interest.

\section{REFERENCES}

1. Papa A, Papa V. The Economic Burden of Diverticular Disease. J Clin Gastroenterol 2016;50 (Suppl 1):S2-S3. doi:10.1097/ MCG.0000000000000598

2. Tursi A. Diverticulosis today: unfashionable and still underresearched. Therap Adv Gastroenterol 2016;9:213-228. doi:10.1177/1756283X15621228

3. Tursi A, Papa A, Danese S. Review article: the pathophysiology and medical management of diverticulosis and diverticular disease of the colon. Aliment Pharmacol Ther 2015;42:664-684. doi:10.1111/apt.13322

4. Garcia G. Diverticulitis. In: Blaser MT, Smith DD, Ravdin JI, eds. Infections of the Gastrointestinal Tract, 2nd ed. Philadelphia: Lippincott Williams \& Wilkins, 2002:306-316.

5. Parks TG. Natural history of diverticular disease of the colon. Clin Gastroenterol 1975;4:53-69.

6. Painter NS, Burkitt DP. Diverticular disease of the colon, a 20th century problem. Clin Gastroenterol 1975;4:3-21. 
7. Strate LL, Erichsen R, Baron JA, et al. Heritability and familial aggregation of diverticular disease: a population-based study of twins and siblings. Gastroenterology 2013;144:736-742. doi:10.1053/j. gastro.2012.12.030

8. Granlund J, Svensson T, Olén $\mathrm{O}$, et al. The genetic influence on diverticular disease - a twin study. Aliment Pharmacol Ther 2012;35:1103-1107. doi:10.1111/j.1365-2036.2012.05069.x

9. Connelly TM, Berg AS, Hegarty JP, et al. The TNFSF15 gene single nucleotide polymorphism rs7848647 is associated with surgical diverticulitis. Ann Surg 2014;259:1132-1137. doi:10.1097/ SLA.0000000000000232

10. Connelly TM, Choi CS, Berg AS, et al. Diverticulitis and Crohn's disease have distinct but overlapping tumor necrosis superfamily 15 haplotypes. J Surg Res 2017;214:262-269. doi:10.1016/j.jss.2017.02.030

11. Coble JL, Sheldon KE, Yue F, et al. Identification of a rare LAMB4 variant associated with familial diverticulitis through exome sequencing. Hum Mol Genet 2017;26:3212-3220. doi:10.1093/hmg/ddx204

12. Reichert MC, Kupcinskas J, Krawczyk M, et al. A variant of COL3A1 (rs3134646) is associated with risk of developing diverticulosis in white men. Dis Colon Rectum 2018;61:604-611. doi:10.1097/ DCR.0000000000001001

13. Sigurdsson S, Alexandersson KF, Sulem P, et al. Sequence variant in ARHGAP15, COLQ and FAM155A associate with diverticular disease and diverticulitis. Nat Commun 2017;8:15789. doi:10.1038/ ncomms15789

14. Maguire LH, Handelman SK, Du X, Chen Y, Pers TH, Speliotes EK Genome-wide association analyses identify 39 new susceptibility loci for diverticular disease. Nat Genet 2018;50:1359-1365. doi:10.1038/ s41588-018-0203-Z

15. Schafmayer C, Harrison JW, Buch S, et al. Genome-wide association analysis of diverticular disease points towards neuromuscular, connective tissue and epithelial pathomechanisms. Gut 2019;68:854865. doi:10.1136/gutjnl-2018-317619

16. Painter NS. Diverticular disease of the colon. Br Med J 1968;3:475-479.

17. Golder M, Burleigh DE, Ghali L, et al. Longitudinal muscle shows abnormal relaxation responses to nitric oxide and contains altered levels of NOS1 and elastin in uncomplicated diverticular disease. Colorectal Dis 2007;9:218-228. doi:10.1111/j.1463-1318.2006.01160.x

18. Parks TG, Connell AM. Motility studies in diverticular disease of the colon. Gut 1969;10:534-542. doi:10.1136/gut.10.7.534

19. Trotman IF, Misiewicz JJ. Sigmoid motility in diverticular disease and the irritable bowel syndrome. Gut 1988;29:218-222. doi:10.1136/ gut.29.2.218

20. Carabotti M, Annibale B, Severi C, Lahner E. Role of fiber in symptomatic uncomplicated diverticular disease: a systematic review. Nutrients 2017;9:E161. doi:10.3390/nu9020161

21. Rao M, Gershon MD. The bowel and beyond: the enteric nervous system in neurological disorders. Nat Rev Gastroenterol Hepatol 2016;13:517-528. doi:10.1038/nrgastro.2016.107

22. Costa M, Brookes SJ, Hennig GW. Anatomy and physiology of the enteric nervous system. Gut 2000;47(Suppl 4):iv15-iv19. doi:10.1136/ gut.47.suppl_4.iv15

23. Ward SM, Sanders KM. Physiology and pathophysiology of the interstitia cell of Cajal: from bench to bedside. I. Functional development and plasticity of interstitial cells of Cajal networks. Am J Physiol Liver Physiol 2001;281:G602-G611. doi:10.1152/ajpgi.2001.281.3.G602

24. Thomsen L, Robinson TL, Lee JC, et al. Interstitial cells of Cajal generate a rhythmic pacemaker current. Nat Med 1998;4:848-851.
25. Bassotti G, Battaglia E, Bellone G, et al. Interstitial cells of Cajal, enteric nerves, and glial cells in colonic diverticular disease. J Clin Pathol 2005;58:973-977. doi:10.1136/jcp.2005.026112

26. Aube AC, Cabarrocas J, Bauer J, et al. Changes in enteric neurone phenotype and intestinal functions in a transgenic mouse model of enteric glia disruption. Gut 2006;55:630-637. doi:10.1136/gut.2005.067595

27. Rao M, Rastelli D, Dong L, et al. Enteric glia regulate gastrointestinal motility but are not required for maintenance of the epithelium in mice. Gastroenterology 2017;153:1068-1081.e7. doi:10.1053/j. gastro.2017.07.002

28. Bär KJ, Facer P, Williams NS, Tam PK, Anand P. Glial-derived neurotrophic factor in human adult and fetal intestine and in Hirschsprung's disease. Gastroenterology 1997;112:1381-1385. doi:10.1016/S0016-5085(97)70154-9

29. Winningham-Major F, Staecker JL, Barger SW, Coats S, Van Eldik LJ. Neurite extension and neuronal survival activities of recombinant S100 beta proteins that differ in the content and position of cysteine residues. J Cell Biol 1989;109:3063-3071. doi:10.1083/jcb.109.6.3063

30. Wedel T, Büsing V, Heinrichs G, et al. Diverticular disease is associated with an enteric neuropathy as revealed by morphometric analysis. Neurogastroenterol Motil 2010;22:407-414. doi:10.1111/j.13652982.2009.01445.X

31. Iwase H, Sadahiro S, Mukoyama S, Makuuchi H, Yasuda M. Morphology of myenteric plexuses in the human large intestine: comparison between large intestines with and without colonic diverticula. J Clin Gastroenterol 2005;39:674-678. doi:10.1097/01.mcg.0000173856.84814.37

32. Barrenschee $M$, Wedel T, Lange $C$, et al. No neuronal loss, but alterations of the GDNF system in asymptomatic diverticulosis. PLoS One 2017;12:e0171416. doi:10.1371/journal.pone.0171416

33. Kim DY, Camilleri M. Serotonin: a mediator of the brain-gut connection. Am J Gastroenterol 2000;95:2698-2709.

34. Galligan JJ, Parkman H. Recent advances in understanding the role of serotonin in gastrointestinal motility and functional bowel disorders. Neurogastroenterol Motil 2007;19(Suppl. 2):1-4. doi:10.11 11/j.13652982.2007.00970.x

35. Böttner M, Barrenschee M, Hellwig I, et al. The enteric serotonergic system is altered in patients with diverticular disease. Gut 2013;62:17531762. doi:10.1136/gutjnl-2012-302660

36. Tursi A. Prucalopride for the Treatment of Symptomatic Uncomplicated Diverticular Disease of the Colon. J Clin Gastroenterol 2016;50:351. doi:10.1097/MCG.0000000000000479

37. Clemens CH, Samsom M, Roelofs J, van Berge Henegouwen GP, Smout AJ. Colorectal visceral perception in diverticular disease. Gut 2004;53:717-722. doi:10.1136/gut.2003.018093

38. Egger B, Peter MK, Candinas D. Persistent symptoms after elective sigmoid resection for diverticulitis. Dis Colon Rectum 2008;51:1044-1048. doi:10.1007/s10350-008-9234-3

39. Humes DJ, Simpson J, Smith J, et al. Visceral hypersensitivity in symptomatic diverticular disease and the role of neuropeptides and low grade inflammation. Neurogastroenterol Motil 2012;24:318-e163. doi:10.1111/j.1365-2982.2011.01863.x

40. Bartho L, Benko R, Holzer-Petsche U, Holzer P, Undi S, Wolf M. Role of extrinsic afferent neurons in gastrointestinal motility. Eur Rev Med Pharmacol Sci 2008;12(Suppl 1):21-31.

41. Pauza AG, Rysevaite-Kyguoliene K, Malinauskas M, et al. Alterations in enteric calcitonin gene-related peptide in patients with colonic diverticular disease: CGRP in diverticular disease. Auton Neurosci 2019;216:63-71. doi:10.1016/j.autneu.2018.09.006 
42. Spiller RC, Humes DJ, Campbell E, et al. The Patient Health Questionnaire 12 Somatic Symptom scale as a predictor of symptom severity and consulting behaviour in patients with irritable bowel syndrome and symptomatic diverticular disease. Aliment Pharmacol Ther 2010;32:811-820. doi:10.1111/j.1365-2036.2010.04402.x

43. Humes DJ, Simpson J, Neal KR, Scholefield JH, Spiller RC. Psychological and colonic factors in painful diverticulosis. Br J Surg 2008;95:195-198. doi:10.1002/bjs.5962

44. Moisset X, Bouhassira D, Denis D, Dominique G, Benoit C, Sabaté JM. Anatomical connections between brain areas activated during rectal distension in healthy volunteers: a visceral pain network. Eur J Pain 2010;14:142-148. doi:10.1016/j.ejpain.2009.04.011

45. Smith JK, Marciani L, Humes DJ, Francis ST, Gowland P, Spiller RC. Anticipation of thermal pain in diverticular disease. Neurogastroenterol Motil 2016;28:900-913. doi:10.1111/nmo.12790

46. Labus JS, Naliboff BD, Berman SM, et al. Brain networks underlying perceptual habituation to repeated aversive visceral stimuli in patients with irritable bowel syndrome. Neuro Image 2009;47:952-960. doi:10.1016/j.neuroimage.2009.05.078

47. Pitiot A, Smith JK, Humes DJ, et al. Cortical differences in diverticular disease and correlation with symptom reports. Neurogastroenterol Motil 2018;30:e13303. doi:10.1111/nmo.13303

48. Painter NS, Burkitt DP. Diverticular disease of the colon: a deficiency disease of western civilization. Br Med J 1971;2:450-454.

49. Aldoori WH, Giovannucci EL, Rockett HR, Sampson L, Rimm EB, Willett WC. A prospective study of dietary fiber types and symptomatic diverticular disease in men. J Nutr 1998;128:714-719. doi:10.1093/ jn/128.4.714

50. Aldoori WH, Giovannucci EL, Rimm EB, Wing AL, Trichopoulos DV, Willett WC. A prospective study of diet and the risk of symptomatic diverticular disease in men. Am J Clin Nutr 1994;60:757-764. doi:10.1093/ajcn/60.5.757

51. Crowe FL, Appleby PN, Allen NE, Key TJ. Diet and risk of diverticular disease in Oxford cohort of European Prospective Investigation into Cancer and Nutrition (EPIC): prospective study of British vegetarians and non-vegetarians. BMJ 2011;343:d4131. doi:10.1136/bmj.d4131

52. Crowe FL, Balkwill A, Cairns BJ, et al. Source of dietary fibre and diverticular disease incidence: a prospective study of UK women. Gut 2014;63:1450-1456. doi:10.1136/gutjnl-2013-304644

53. Strate LL, Liu YL, Syngal S, Aldoori WH, Giovannucci EL. Nut, corn, and popcorn consumption and the incidence of diverticular disease. JAMA 2008;300:907-914. doi:10.1001/jama.300.8.907

54. Maguire LH, Song M, Strate LE, Giovannucci EL, Chan AT. Higher serum levels of vitamin D are associated with a reduced risk of diverticulitis. Clin Gastroenterol Hepatol 2013;11:1631-1635. doi:10.1016/j.cgh.2013.07.035

55. Cao Y, Strate LL, Keeley BR, et al. Meat intake and risk of diverticulitis among men. Gut 2018;67:466-472. doi:10.1136/gutjnl-2016-313082

56. Peery AF, Barrett PR, Park D, et al. A high-fiber diet does not protect against asymptomatic diverticulosis. Gastroenterology 2012;142:266272.e1. doi:10.1053/j.gastro.2011.10.035

57. Peery AF, Sandler RS, Ahnen DJ, et al. Constipation and a low-fiber diet are not associated with diverticulosis. Clin Gastroenterol Hepatol 2013;11:1622-1627. doi:10.1016/j.cgh.2013.06.033

58. Tursi A. Dietary pattern and colonic diverticulosis. Curr Opin Clin Nutr Metab Care 2017;20:409-413. doi:10.1097/MCO.0000000000000403

59. Sawicki CM, Livingston KA, Obin M, Roberts SB, Chung M, McKeown NM. Dietary fiber and the human gut microbiota: application of evidence mapping methodology. Nutrients 2017;9:E125. doi:10.3390/ nu9020125

60. Maguire LH, Song M, Strate LL, Giovannucci EL, Chan AT. Association of geographic and seasonal variation with diverticulitis admissions. JAMA Surg 2015;150:74-77. doi:10.1001/jamasurg.2014.2049

61. Jamal Talabani A, Lydersen S, Ness-Jensen E, Endreseth BH, Edna TH. Risk factors of admission for acute colonic diverticulitis in a populationbased cohort study:the North Trondelag Health Study, Norway. World J Gastroenterol 2016;22:10663-10672. doi:10.3748/wjg.v22.i48.10663

62. Turunen P, Wikström H, Carpelan-Holmström M, Kairaluoma P, Kruuna O, Scheinin T. Smoking increases the incidence of complicated diverticular disease of the sigmoid colon. Scand J Surg 2010;99:14-17. doi:10.1177/145749691009900104

63. Hjern F, Wolk A, Håkansson N. Smoking and the risk of diverticular disease in women. Br J Surg 2011;98:997-1002. doi:10.1002/bjs.7477

64. Humes DJ, Fleming KM, Spiller RC, West J. Concurrent drug use and the risk of perforated colonic diverticular disease:a populationbased case-control study. Gut 2011;60:219-224. doi:10.1136/ gut.2010.217281

65. Strate LL, Liu YL, Aldoori WH, Syngal S, Giovannucci EL. Obesity increases the risks of diverticulitis and diverticular bleeding. Gastroenterology 2009;136:115-122.e1. doi:10.1053/j. gastro.2008.09.025

66. Strate LL, Liu YL, Aldoori WH, Giovannucci EL. Physical activity decreases diverticular complications. Am J Gastroenterol 2009;104:1221-1230.

67. Aldoori WH, Giovannucci EL, Rimm EB, Wing AL, Trichopoulos DV, Willett WC. A prospective study of alcohol, smoking, caffeine, and the risk of symptomatic diverticular disease in men. Ann Epidemiol 1995;5:221-228. doi:10.1016/1047-2797(94)00109-7

68. Nagata N, Niikura R, Shimbo T, et al. Alcohol and smoking affect risk of uncomplicated colonic diverticulosis in Japan. PLoS One 2013;8:e81137. doi:10.1371/journal.pone.0081137

69. Aldoori WH, Giovannucci EL, Rimm EB, Wing AL, Willett WC. Use of acetaminophen and nonsteroidal anti-inflammatory drugs:a prospective study and the risk of symptomatic diverticular disease in men. Arch Fam Med 1998;7:255-260.

70. Strate LL, Liu YL, Huang ES, Giovannucci EL, Chan AT. Use of aspirin or nonsteroidal anti-inflammatory drugs increases risk for diverticulitis and diverticular bleeding. Gastroenterology 2011;140:1427-1433. doi:10.1053/j.gastro.2011.02.004

71. Broersen LHA, Horváth-Puhó E, Pereira AM, Erichsen R, Dekkers OM, Sørensen HT. Corticosteroid use and mortality risk in patients with perforated colonic diverticular disease: a population-based cohort study. BMJ Open Gastroenterol 2017;4:e000136. doi:10.1136/ bmjgast-2017-000136

72. Morris CR, Harvey IM, Stebbings WS, Speakman CT, Kennedy HJ Hart AR. Do calcium channel blockers and antimuscarinics protect against perforated colonic diverticular disease? A case control study. Gut 2003;52):1734-1737. doi:10.1136/gut.52.12.1734

73. Belkaid Y, Harrison OJ. Homeostatic immunity and the microbiota Immunity 2017;46:562-576. doi:10.1016/j.immuni.2017.04.008

74. Kumar H, Kawai T, Akira S. Pathogen recognition in the innate immune response. Biochem J 2009;420:1-16. doi:10.1042/BJ20090272

75. Akira S, Uematsu S, Takeuchi O. Pathogen recognition and innate immunity. Cell 2006;124:783-801. doi:10.1016/j.cell.2006.02.015

76. Peery AF, Keku TO, Addamo C, et al. Colonic diverticula are not associated with mucosal inflammation or chronic gastrointestinal 
symptoms. Clin Gastroenterol Hepatol 2018;16:884-891.e1. doi:10.1016/j.cgh.2017.05.051

77. Von Rahden BH, Kircher S, Thiery S, et al. Association of steroid use with complicated sigmoid diverticulitis:potential role of activated CD68+/CD163+ macrophages. Langenbeck's Arch Surg 2011;396:759768. doi:10.1007/s00423-011-0797-4

78. Barbara G, Scaioli E, Barbaro MR, et al. Gut microbiota, metabolome and immune signatures in patients with uncomplicated diverticular disease. Gut 2016;66:1252-1261. doi:10.1136/gutjnl-2016-312377

79. Narayan R, Floch MH. Microscopic colitis as part of the natural history of diverticular disease. Am J Gastroenterol 2002;97:S112-S113. doi:10.1016/S0002-9270(02)04814-1

80. Tursi A, Brandimarte G, Elisei W, et al. Assessment and grading of mucosal inflammation in colonic diverticular disease. J Clin Gastroenterol 2008;42:699-703. doi:10.1097/MCG.0b013e3180653ca2

81. Braegger CP, Nicholls S, Murch SH, Stephens S, MacDonald TT. Tumour necrosis factor alpha in stool as a marker of intestinal inflammation. Lancet 1992;339:89-91. doi:10.1016/0140-6736(92)90999-J

82. Plevy SE, Landers CJ, Prehn J, et al. A role for TNF-alpha and mucosal Thelper-1 cytokines in the pathogenesis of Crohn's disease. J Immunol 1997;159:6276-6282.

83. Berner B, Akca D, Jung T, Muller GA, Reuss-Borst MA. Analysis of Th1 and Th2 cytokines expressing CD4+ and CD8+ T cells in rheumatoid arthritis by flow cytometry. J Rheumatol 2000;27:1128-1135.

84. Tursi A, Elisei W, Brandimarte G, et al. Mucosal tumour necrosis factor-alpha in diverticular disease of the colon is overexpressed with disease severity. Colorectal Dis 2012;14:e258-e263. doi:10.1111/j.14631318.2012.02926.x

85. Humes DJ, Simpson J, Smith J, et al. Visceral hypersensitivity in symptomatic diverticular disease and the role of neuropeptides and low grade inflammation. Neurogastroenterol Motil 2012;24:318-e163. doi:10.1111/j.1365-2982.2011.01863.x

86. Tursi A, Elisei W, Giorgetti GM, et al. Expression of basic fibroblastic growth factor, syndecan 1 and tumour necrosis factor $\alpha$ in resected acute colonic diverticulitis. Colorectal Dis 2014;16:O98-O103. doi:10.1111/ codi. 12504

87. Neurath MF. Cytokines in inflammatory bowel disease. Nat Rev Immunol 2014;14:329-342. doi:10.1038/nri3661

88. Turco F, Andreozzi P, Palumbo I, et al. Bacterial stimuli activate nitric oxide colonic mucosal production in diverticular disease. Protective effects of L. casei DG (Lactobacillus paracasei CNCM I-1572). United European Gastroenterol J 2017;5:715-724. doi:10.1177/2050640616684398

89. Tursi A, Mastromarino P, Capobianco D, Elisei W, Picchio M, Brandimarte G. No changes in Interleukin-10 expression in symptomatic uncomplicated diverticular disease of the colon. J Gastrointestin Liver Dis 2018;27:476-477. doi:10.15403/jgld.2014.1121.274.ink

90. Dai L, King DW, Perera DS, Lubowski DZ, Burcher E, Liu L. Inverse expression of prostaglandin E2-related enzymes highlights differences between diverticulitis and inflammatory bowel disease. Dig Dis Sci 2015;60:1236-1246. doi:10.1007/s10620-014-3478-7

91. Schieffer KM, Choi CS, Emrich S, et al. RNA-seq implicates deregulation of the immune system in the pathogenesis of diverticulitis. Am J Physiol Gastrointest Liver Physiol 2017;313:G277-G284. doi:10.1152/ ajpgi.00136.2017

92. Cossais F, Leuschner S, Barrenschee M, et al. Persistent Increased Enteric Glial Expression of S100 $\beta$ is Associated With Low-grade Inflammation in Patients With Diverticular Disease. J Clin Gastroenterol 2018 Mar 6. doi:10.1097/MCG.0000000000001011.
93. Johansson MEV, Larsson JM, Hansson GC. The two mucus layers of colon are organized by the MUC2 mucin, whereas the outer layer is a legislator of host-microbial interactions. Proc Natl Acad Sci U S A 2011;108(Suppl 1):4659-4665. doi:10.1073/pnas.1006451107

94. Johansson ME, Phillipson M, Petersson J, Velcich A, Holm L, Hansson GC. The inner of the two Muc2 mucin-dependent mucus layers in colon is devoid of bacteria. Proc Natl Acad Sci U S A 2008;105:15064-15069. doi:10.1073/pnas.0803124105

95. Tollin M, Bergman P, Svenberg T, Jörnvall H, Gudmundsson GH, Agerberth B. Antimicrobial peptides in the first line defence of human colon mucosa. Peptides 2003;24:523-530. doi:10.1016/S01969781(03)00114-1

96. Eckburg PB, Bik EM, Bernstein CN, et al. Diversity of the human intestinal microbial flora. Science 2005;308:1635-1638. doi:10.1126/ science. 1110591

97. Faith JJ, Guruge JL, Charbonneau M, et al. The long-term stability of the human gut microbiota. Science 2013;341:1237439. doi:10.1126/ science. 1237439

98. Human Microbiome Project Consortium. Structure, function and diversity of the healthy human microbiome. Nature 2012;486:207-214. doi:10.1038/nature11234

99. Matsuoka K, Kanai T. The gut microbiota and inflammatory bowel disease. Semin Immunopathol 2015;37:47-55. doi:10.1007/s00281014-0454-4

100. Strate LL, Modi R, Cohen E, Spiegel BM. Diverticular disease as a chronic illness:evolving epidemiologic and clinical insights. Am J Gastroenterol 2012;107:1486-1493. doi:10.1038/ajg.2012.194

101. Machiels K, Joossens M, Sabino J, et al. A decrease of the butyrateproducing species Roseburia hominis and Faecalibacterium prausnitzii defines dysbiosis in patients with ulcerative colitis. Gut 2014;63:12751283. doi:10.1136/gutjnl-2013-304833

102. Ponziani FR, Scaldaferri F, Petito V, et al. The Role of Antibiotics in Gut Microbiota Modulation: The Eubiotic Effects of Rifaximin. Dig Dis 2016;34:269-278. doi:10.1159/000443361

103. Png CW, Lindén SK, Gilshenan KS, et al. Mucolytic bacteria with increased prevalence in IBD mucosa augment in vitro utilization of mucin by other bacteria. Am J Gastroenterol 2010;105:2420-2428. doi:10.1038/ajg.2010.281

104. Tursi A, Mastromarino P, Capobianco D, et al. Assessment of fecal microbiota and fecal metabolome in symptomatic uncomplicated diverticular disease of the colon. J Clin Gastroenterol 2016;50(Suppl 1):S9-S12. doi:10.1097/MCG.0000000000000626

105. Laghi L, Mastromarino P, Elisei W, et al. Impact of treatments on fecal microbiota and fecal metabolome in symptomatic uncomplicated diverticular disease of the colon: a pilot study. J Biol Regul Homeost Agents 2018;32:1421-1432.

106. Rooijers K, Kolmeder C, Juste C, et al. An iterative workflow for mining the human intestinal metaproteome. BMC Genomics 2011;12:6. doi:10.1186/1471-2164-12-6

107. Derrien M, Vaughan EE, Plugge CM, de Vos WM. Akkermansia muciniphila gen. nov., sp. nov., a human intestinal mucin-degrading bacterium. Int J Syst Evol Microbiol 2004;54:1469-1476. doi:10.1099/ ijs.0.02873-0

108. Biagi E, Nylund L, Candela M, et al. Through ageing, and beyond: gut microbiota and inflammatory status in seniors and centenarians. PLoS One 2010;5:e10667. doi:10.1371/journal.pone.0010667

109. Daniels L, Budding AE, de Korte N, et al. Fecal microbiome analysis as a diagnostic test for diverticulitis. Eur J Clin Microbiol Infect Dis 2014;33:1927-1936. doi:10.1007/s10096-014-2162-3 
110. Schieffer KM, Sabey K, Wright JR, et al. The microbial ecosystem distinguishes chronically diseased tissue from adjacent tissue in the sigmoid colon of chronic, recurrent diverticulitis patients. Sci Rep 2017;7:8467. doi:10.1038/s41598-017-06787-8

111. Lupp C, Robertson ML, Wickham ME, et al. Host-mediated inflammation disrupts the intestinal microbiota and promotes the overgrowth of Enterobacteriaceae. Cell Host Microbe 2007;2:119-129. doi:10.1016/j.chom.2007.06.010

112. Kvasnovsky CL, Leong LEX, Choo JM, et al. Clinical and symptom scores are significantly correlated with fecal microbiota features in patients with symptomatic uncomplicated diverticular disease: a pilot study. Eur J Gastroenterol Hepatol 2018;30:107-112. doi:10.1097/ MEG.0000000000000995

113. Cui L, Morris A, Ghedin E. The human mycobiome in health and disease. Genome Med 2013;5:63. doi:10.1186/gm467

114. Hallen-Adams HE, Kachman SD, Kim J, Legge RM, Martinez I. Fungi inhabiting the healthy human gastrointestinal tract:a diverse and dynamic community. Fungal Ecol 2015;15:9-17. doi:10.1016/j. funeco.2015.01.006
115. Hallen-Adams HE, Suhr MJ. Fungi in the healthy human gastrointestinal tract. Virulence 2017;8:352-358. doi:10.1080/21505 594.2016.1247140

116. Dollive S, Chen YY, Grunberg S, et al. Fungi of the murine gut: episodic variation and proliferation during antibiotic treatment. PLoS One 2013;8:e71806. doi:10.1371/journal.pone.0071806

117. Carding SR, Davis N, Hoyles L. Review article: the human intestinal virome in health and disease. Aliment Pharmacol Ther 2017;46:800-815. doi:10.1111/apt.14280

118. Lopetuso LR, Ianiro G, Scaldaferri F, Cammarota G, Gasbarrini A Gut Virome and Inflammatory Bowel Disease. Inflamm Bowel Dis 2016;22:1708-1712. doi:10.1097/MIB.0000000000000807

119. Hollink N, Dzabic M, Wolmer N, Boström L, Rahbar A. High prevalence of an active human cytomegalovirus infection in patients with colonic diverticulitis. J Clin Virol 2007;40:116-119. doi:10.1016/j.jcv.2007.07.008

120. Schieffer KM, Kline BP, Harris LR, Deiling S, Koltun WA, Yochum GS A differential host response to viral infection defines a subset of earlieronset diverticulitis patients. J Gastrointestin Liver Dis 2018;27:249-255. doi:10.15403/jgld.2014.1121.273.sch 